\title{
The mTOR pathway activity in ASD and post-infectious neuropsychiatric autoimmune disorders
}

\author{
Ekaterina A. Trifonova \\ Gene Engineering Laboratory \\ Institute of Cytology and Genetics \\ SB RAS \\ Novosibirsk, Russia \\ trifonova.k@rambler.ru \\ Zakhar S. Mustafin \\ Laboratory of Molecular Genetic \\ Systems \\ Institute of Cytology and Genetics \\ SB RAS \\ Novosibirsk, Russia \\ mustafinZS@bionet.nsc.ru
}

\author{
Alexandra I. Klimenko \\ Laboratory of Molecular Genetic \\ Systems \\ Institute of Cytology and Genetics \\ SB RAS \\ Novosibirsk, Russia \\ klimenko@bionet.nsc.ru
}

Sergey A. Lashin
Laboratory of Molecular Genetic
Systems
Institute of Cytology and Genetics
SB RAS
Novosibirsk, Russia
lashin@bionet.nsc.ru

\author{
Sviatoslav L. Bezrodny \\ Laboratiry of \\ Bifidobacteria Biology \\ Institute of Epidemiology \& \\ Microbiology \\ Moscow, Russia \\ frebiotik@mail.ru
}

Alex V. Kochetov

Gene Engineering Laboratory

Institute of Cytology and Genetics SB RAS

Novosibirsk, Russia

ak@bionet.nsc.ru

\begin{abstract}
Autism spectrum disorder (ASD) has a strong and complex genetic component with an estimate of more than 1000 genes implicated cataloged in SFARI (Simon's Foundation Autism Research Initiative) Gene database. We conducted geneset analyses and revealed that 179 out of 281 genes (64\%) included in the first three categories of the database ("High confidence", "Strong candidate", and "Suggestive evidence") can be attributed to one of the four groups: 1. FMRP target genes, 2. mTOR signaling network genes, 3. mTOR-modulated genes, 4. Vitamin D3 sensitive genes. The additional gene network analysis revealed 43 new genes and 127 new interactions so in the whole 222 out of $281(79 \%)$ high scored genes from SFARI Gene database are connected with mTOR signaling activity and/or dependent on vitamin D3 availability directly or indirectly. Infections and the resulting immune response to these infections have recently been increasingly recognized as pathogenic mechanism for neuropsychiatric disorders. Sydenham's Chorea (SC), a widely recognized poststreptococcal autoimmune disease, provides a model for studying the disorders. Significant overlap of ASD and SC/PANS/PANDAS symptoms makes complicated both differential diagnosis and search for the common molecular mechanisms underlying the disorders. We analyzed bioinformatically the small intestine microbiome obtained by mass spectrometry of microbial markers in the Russian population of patients with ASD. As a result, quantitative data on the percentage of patients with significantly elevated levels of Streptococcus spp and Clostridium spp in this population were first obtained.
\end{abstract}

Keywords - autism spectrum disorders (ASD), Sydenham's Chorea (SC), PANDAS (pediatric autoimmune neuropsychiatric disorder associated with streptococcal infections), PANS (pediatric autoimmune neuropsychiatric disorder), mTOR (mechanistic target of rapamycin)

\section{Introduction}

Autism spectrum disorder (ASD) is a heterogeneous neurodevelopmental disorder with complex genetic, environmental, and epigenetic components. The search for genetic factors underlying ASD has led to the identification of more than one thousand genes cataloged in SFARI (Simon's Foundation Autism Research Initiative) Gene database that has scored and ranked genes into one of the seven categories [1]. A significant part of both syndromic and idiopathic autism cases can be attributed to disorders caused by mTORdependent translation deregulation. Protein biosynthesis, or translation, is a finely regulated process, with the central role played by mTOR kinase (mechanistic target of rapamycin). A mutational aberration in at least one of the links of the mTOR signaling pathway impairs the synaptic plasticity and behavior. The deregulation of local translation in dendrites is connected with the following monogenic ASDs: neurofibromatosis type 1, Noonan syndrome, Costello syndrome, Cowden syndrome, tuberous sclerosis, fragile $\mathrm{X}$ syndrome, and Rett syndrome [2].

\section{Results and Discussion}

We conducted gene-set analyses and revealed that 179 out of 281 genes $(64 \%)$ included in the first three categories of the database ("High confidence", "Strong candidate", and "Suggestive evidence") can be attributed to one of the four groups: 1. FMRP target genes, 2. mTOR signaling network genes, 3. mTOR-modulated genes, 4. Vitamin D3 sensitive genes. The additional gene network analysis revealed 43 new genes and 127 new interactions so in the whole 222 out of 281 (79\%) high scored genes from SFARI Gene database are connected with mTOR signaling activity and/or dependent on vitamin D3 availability directly or indirectly. A significant portion of genes that belong to more than one of four categories was of particular interest (Table 1).

TABLE 1. HIGH SCORED GENES IMPLICATED IN AUTISM SUSCEPTIBILITY THAT FALL INTO MORE THAN ONE CATEGORY

\begin{tabular}{|l|l|l|}
\hline Categories of genes & $\begin{array}{l}\text { Total } \\
\text { number }\end{array}$ & Elements \\
\hline $\begin{array}{l}\text { FMRP target } \cap \text { Vitamin } \mathrm{D} \\
\text { sensitive } \cap \text { mTOR signaling } \\
\text { pathway } \cap \text { mTOR-modulated }\end{array}$ & 1 & PTEN \\
\hline $\begin{array}{l}\text { FMRP target } \cap \text { mTOR } \\
\text { signaling pathway } \cap \text { mTOR- } \\
\text { modulated }\end{array}$ & 4 & $\begin{array}{l}\text { SYNGAP1 CUL7 TSC2 } \\
\text { CTNNB1 }\end{array}$ \\
\hline
\end{tabular}




\begin{tabular}{|c|c|c|}
\hline $\begin{array}{l}\text { FMRP target } \cap \text { Vitamin } D \\
\text { sensitive } \cap \text { mTOR-modulated }\end{array}$ & 4 & $\begin{array}{l}\text { SMARCC2 ANKRD11 } \\
\text { KDM5C CLASP1 }\end{array}$ \\
\hline $\begin{array}{l}\text { Vitamin D sensitive } \cap \text { mTOR } \\
\text { signaling pathway } \cap \text { mTOR- } \\
\text { modulated }\end{array}$ & 1 & $\mathrm{CCT} 4$ \\
\hline $\begin{array}{l}\text { FMRP target } \cap \text { mTOR- } \\
\text { modulated }\end{array}$ & 29 & $\begin{array}{l}\text { EP400 USP7 UBR5 PHRF1 } \\
\text { GGNBP2 UBE3C KMT2E } \\
\text { CHD8 AKAP9 ATP2B2 } \\
\text { PLXNB1 MED13 LEO1 } \\
\text { DPYSL2 KAT6A TRIP12 } \\
\text { DYNC1H1 STXBP1 EHMT1 } \\
\text { MYH10 AGAP2 CUX1 } \\
\text { TAOK2 ANK3 SETD5 } \\
\text { SLC12A5 CC2D1A } \\
\text { TNRC6B NCKAP1 }\end{array}$ \\
\hline $\begin{array}{l}\text { FMRP target } \cap \text { mTOR } \\
\text { signaling pathway }\end{array}$ & 2 & MTOR PRKCB \\
\hline $\begin{array}{l}\text { FMRP target } \cap \text { Vitamin } D \\
\text { sensitive }\end{array}$ & 15 & $\begin{array}{l}\text { NUAK1 SMARCA4 } \\
\text { MYO9B SHANK3 MED13L } \\
\text { ARID1B DLGAP1 } \\
\text { SPARCL1 TCF20 WDFY3 } \\
\text { KCNQ3 SBF1 TCF4 } \\
\text { PRICKLE2 HIVEP3 }\end{array}$ \\
\hline $\begin{array}{l}\text { Vitamin D sensitive } \cap \text { mTOR- } \\
\text { modulated }\end{array}$ & 10 & $\begin{array}{l}\text { RAB2A ELP4 PHB } \\
\text { PRICKLE1 BCKDK NAA15 } \\
\text { ASTN2 ETFB CAPRIN1 } \\
\text { SLC35B1 }\end{array}$ \\
\hline $\begin{array}{l}\text { Vitamin D sensitive } \cap \text { mTOR } \\
\text { signaling pathway }\end{array}$ & 2 & DYRK1A SLC7A5 \\
\hline
\end{tabular}

We have hypothesized that genetic and/or environment mTOR hyperactivation including provoked by vitamin D deficiency might be a common mechanism controlling expressivity of most autism predisposition genes and even core symptoms of autism.

Infections and the resulting immune response to these infections have recently been increasingly recognized as pathogenic mechanism for neuropsychiatric disorders.
Sydenham's Chorea (SC), a widely recognized poststreptococcal autoimmune disease, provides a model for studying the disorders. PANDAS (pediatric autoimmune neuropsychiatric disorder associated with streptococcal infections) has been proposed as a variant of SC, so that they have a common pathogenesis, despite the unique profile of the predominantly psychiatric symptoms of PANDAS [3]. It should be noted that streptococcus spp are not unique in their ability to cause autoimmune neuropsychiatric complications; among alternative inducers, influenza and chickenpox viruses, mycoplasmas, in general, the entire spectrum of such syndromes is called PANS.

Significant overlap of ASD and SC/PANS/PANDAS symptoms makes complicated both differential diagnosis and search for the common molecular mechanisms underlying the disorders. We analyzed bioinformatically the small intestine microbiome obtained by mass spectrometry of microbial markers in the Russian population of patients with ASD. As a result, quantitative data on the percentage of patients with significantly elevated levels of Streptococcus spp and Clostridium spp in this population were first obtained.

It was recently shown that mTORC1 is a central pathway in the pathogenesis of systemic lupus erythematosus and other autoimmune diseases [4]. At the same time, a significant percentage of ASD is associated with activation of the mTORC1 signaling pathway. The causal relationship between mTORC1 activation, an increased level of pathogenic microflora in the intestines of patients with ASD, and autoimmune syndromes often found in ASD should be the subject of future research.

\section{ACKNOWLEDGMENT}

This research was supported by the Russian State Budget (project No. 0259-2019-0008).

\section{REFERENCES}

[1] B. S. Abrahams, et al SFARI Gene 2.0: A community-driven knowledgebase for the autism spectrum disorders (ASDs). Mol. Autism. 2013, vol. 4, pp. 36-39.

[2] J.O. Lipton, M. Sahin. The Neurology of mTOR. Neuron. 2014, vol. 84, pp. 275-291.

[3] K.A. Williams, S.E. Swedo. Post-infectious autoimmune disorders: Sydenham's chorea, PANDAS and beyond. Brain Res. 2015 vol. 1617, pp. 144-54.

[4] T. Suto, T. Karonitsch. The immunobiology of mTOR in autoimmunity. J Autoimmun., in press. 